\title{
Opportunistic Usage of Maritime VHF Band-Deployment Challenges for a New Regulatory Framework
}

\author{
Eduardo Bolas $^{1,2}$, Nuno Borges de Carvalho ${ }^{1}$, José Neto Vieira ${ }^{3}$, Paulo Mónica de Oliveira ${ }^{2}$ \\ ${ }^{1}$ Instituto de Telecomunicações/DETI, Universidade de Aveiro, Aveiro, Portugal; ${ }^{2}$ Naval Research Centre (CINAV)-Escola Naval, \\ Almada, Portugal; ${ }^{3}$ Instituto de Engenharia Electrónica e Telemática de Aveiro/DETI, Universidade de Aveiro, Aveiro, Portugal. \\ Email: eduardobolas@ua.pt
}

Received October $3^{\text {rd }}, 2013$; revised November $13^{\text {th }}, 2013$; accepted November $29^{\text {th }}, 2013$

Copyright (C) 2014 Eduardo Bolas et al. This is an open access article distributed under the Creative Commons Attribution License, which permits unrestricted use, distribution, and reproduction in any medium, provided the original work is properly cited. In accordance of the Creative Commons Attribution License all Copyrights (C) 2014 are reserved for SCIRP and the owner of the intellectual property Eduardo Bolas et al. All Copyright (C) 2014 are guarded by law and by SCIRP as a guardian.

\section{ABSTRACT}

Similarly to other domains, maritime community requests for broadband services have been significantly increasing. Worldwide navigation footprint and the lack of practical alternatives to Satellite Communications (SATCOM) empower VHF band as the natural choice to support most of those demands. Nevertheless, the major challenge for an implementation of maritime broadband VHF services is unquestionably the spectrum availability and management. Eventually, the solution must include spectrum sharing, using a Cognitive Radio (CR) based approach, but unfortunately current regulatory framework and spectrum management regime are not appropriate for such concepts and emerging technologies. To overcome such constraints, it is necessary to address a whole field of regulatory and standardization issues in order to prepare an evolution towards a more flexible and dynamic approach to spectrum management and a transition that would ensure incumbents live operations and legacy systems. The required paradigm change encompasses a new policy definition, an enforcement mechanism implementation and a comprehensive transition plan. The presented analysis pretends to address the regulatory feasibility of a framework change, discusses its evolving process and points some challenges related with practical aspects associated to Quality of Service (QoS) enforcement metrics definition, centering the arguments in maritime VHF band.

\section{KEYWORDS}

\section{Maritime Communications; Broadband VHF; Cognitive Radio; Regulatory Framework; Dynamic Spectrum} Access; Metrics

\section{Introduction}

The tremendous developments in services and applications have unquestionable impact on performance requirements at network level. Independently of transmission media, user demands have been increased significantly and the need for broadband services is a common issue across the spectrum. The maritime community is no exception. From emerging maritime safety related concepts, such as e-navigation, to Internet access for cruisers' passengers, the need for broadband services at sea is an important operational requirement that, currently, has serious constraints at performance and budget levels. Maritime radio-communications supported services are restricted to voice and low rate data like Digital Selective Calling (DSC) and potential alternatives like Universal Mobile Telecommunications System (UMTS) or Long Term Evolution (LTE) networks have very limited sea coverage. Therefore, when data services are required, SATCOM is the preferable choice for most mariners, despite shore proximity, performance and associated costs. This situation is obviously unacceptable and cannot be sustained much longer, especially because radio-communications state of the art allows solutions that would potentially change the current maritime communications paradigm.

Interesting enough, a significant world sailing activity occurs within 40 miles from coast line, as presented in 
the example of Figure 1. Besides the regional and local traffic, mostly constituted by fishing vessels and recreational boats, international cruisers, cargo ships and tankers plan their journeys as close as possible to shore. Under those conditions, a shore based VHF system able to provide attractive network based services for mariners, would represent a significant improvement in maritime communications and a potential business opportunity with positive impact in different domains.

However, the major challenge for an implementation of broadband services in maritime VHF band is unquestionably the spectrum availability and management. It is difficult to find and assign spectrum for new services, especially contiguous spectra. A possible solution might be an integration of capabilities, based on new emerging concepts and technologies, such as CR, multi-carrier modulation techniques and smart antennas, and apply them to maritime VHF band in order to take advantage of spectrum opportunities, enabling a spectrally efficient and high-speed data communications system. Nevertheless, the current regulatory framework is not prepared for emerging radio communications technologies and governing issues are still an active area of discussions. On the other hand, spectrum underutilization and scarcity are incontestable facts, intensifying a common understanding that Dynamic Spectrum Access (DSA) is the only strategy to overcome identified problems. In any case, there is an obvious need for an evolution on regulatory framework, not only because current model does not promote efficient use of spectrum, but also because it cannot support other vectors of regulatory mission, namely the detachment of barriers throughout telecommunications sector and the support of business.

The objective of the presented paper is to address possible ways to evolve regulatory framework towards a more flexible and dynamic management process, centring the discussion on regulatory and standardization issues associated to the application of CR concepts to ma-

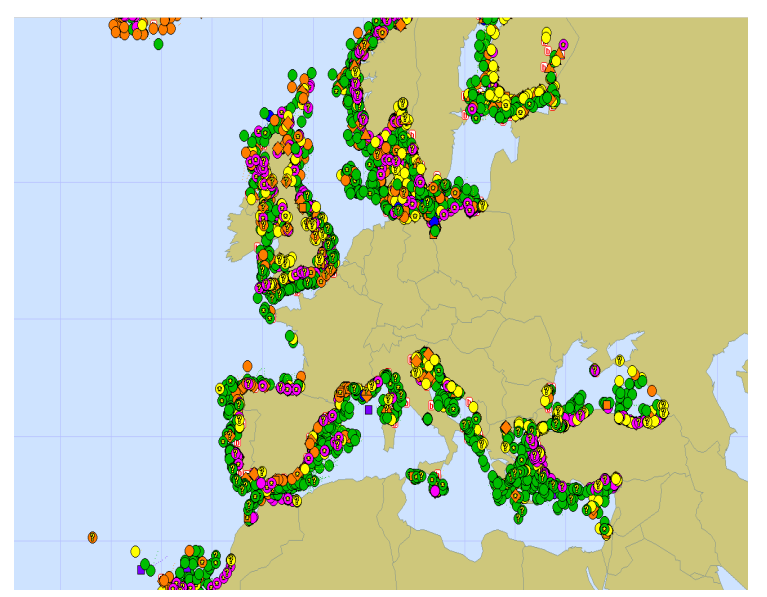

Figure 1. A snapshot of automatic identification system information, where each color dot represents a vessel. ritime VHF band. Practical aspects, related with Quality of Service (QoS) enforcement to ensure incumbents live operations and legacy systems coexistence, namely figures of merit, are also tackled. Obviously, this subject is too complex to be wrapped with this exercise, but we expect to contribute for on-going discussions, which, hopefully, will enable VHF based maritime broadband services in a near future.

The remainder of the paper is organized as follows: in Section II we discuss the role of CR in spectrum scarcity mitigating processes, namely in the support of DSA; Section III is dedicated to the challenges and opportunities associated to an evolution on regulatory framework and Section IV addresses the practical aspects related with the deployment of a spectrum allocation strategy, namely operational caveats such as QoS enforcement, metrics and equipment certification. Final remarks are presented in Section V.

\section{Cognitive Radio-The Catalyst of DSA}

In the past, the fear of harmful interference led to static allocation strategies, which have been largely successful in the protection of assigned users, but poorly efficient in terms of overall spectrum utilization. Typically, the frequency bands are assigned to a specific user or service, which are guaranteed to have exclusive access to that portion of spectrum, no matter how often it is used. As a result, static strategy has been largely successful in the protection of assigned users, but poorly efficient in terms of overall spectrum utilization. In the case of Maritime Mobile Service (MMS), there are frequency bands assign to particular users and to specific purpose, namely channel 16, which is assigned for distress, safety and calling voice services. Figure 2 presents a spectrogram for MMS allocated band where the inefficiency of spectrum occupancy is incontestable. Hence, any initiative to im-

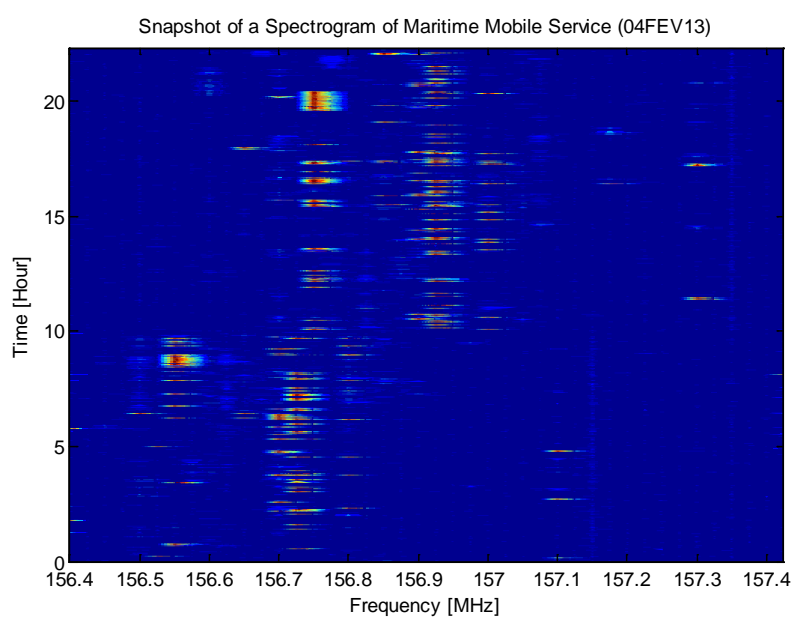

Figure 2. Spectrogram of MMS within Lisbon line-of-sight area (including port approach and Tagus River estuary). 
prove the efficiency, within actual frameworks, would involve a re-evaluation of demands and reassignment of spectrum, which is obviously unfeasible due to natural constraints associated to live operations and legal issues. However, current spectrum availability difficulties claim for new approaches to management, more flexible, adaptable and efficient. Alternatively, a dynamic approach, such as DSA, where the spectrum access is not restricted, has a potential for flexible and optimal management. Nevertheless, the potential risk of harmful interference is significantly higher, than in static case, which sometimes results in mistrust on dynamic model adequacy to support live operations. That is why the deployment of a new spectrum management paradigm needs to be associated to the benefits of static spectrum access, concerning interference and coexistence of systems with different precedence.

The opportunistic usage of spectrum is probably the most important endeavour to overcome spectrum scarcity. Even though, DSA addresses the fundamental issues and enable feasible implementations of required features, it cannot be appointed as a spectrum access policy without the technological support to enable governance models deployment. This is particularly important, not only to provide a reliable solution to handle radio environment challenges and opportunities, but also to offer an unquestionable answer to all spectrum stakeholders, regarding its capacity to deal with interference and assure live operations requirements. In fact, the key for spectrum efficient usage does not reside on management strategy definition merit, but on the quality and possibility to implement it.

The principles of DSA are associated to the need of taking advantage of inactive spectrum segments through opportunistic access to radio spectrum bands that are not being used. Curiously, Mitola [1] proposed the concept of CR, which is exactly an approach to increase spectrum efficiency exploring frequency bands capacity to accommodate non-licensed transmissions (secondary users), without harming operation of incumbents (primary users). According to Federal Communications Commission (FCC) [2], CR is "a radio or system that senses its operational electromagnetic environment and can dynamically and autonomously adjust its radio operating parameters to modify system operation, such as maximize throughput, mitigate interference, facilitate interoperability, access secondary markets." The development of CR concept presents an extraordinary opportunity to implement a set of functionalities that are vital for opportunistic usage of spectrum and consequently decisive to increase the efficiency of electromagnetic radio spectrum usage and overcome its scarcity.

Naturally, the expected efficiency improvement is proportional to the level of cognition functionalities available in the implementation of a CR. This means that a CR ability to exploit inactive frequency bands will depend upon its faculty to find the so-called opportunities to operate and consequently on its spectrum sensing techniques capabilities. Therefore, one cannot think in $\mathrm{CR}$ as a silver bullet for all the problems, not only because the technology is not mature enough, but also because the radio communications stakeholders are not ready for the application of such disruptive mind set approach. Anyway, the discussion shall not be centred in the problem, but focused on the solution. In other words, the recurrent issue is not the spectrum scarcity, but the lack of capacity to implement an efficient and effective DSA scheme. Apparently, CR represents the searched aptitude to successfully implement DSA, so the empowering of its deployment should be also on governance and policy side.

\section{Evolution of Regulatory Framework}

The regulatory framework for radio spectrum management has two basic processes: allocation and assignment. The spectrum allocation is an international cooperative process where frequency bands are devoted to a specific use or service. The frequency assignment, mainly a National Regulatory Authority (NRA) role, is a licensing process of spectrum to a specific user or purpose. Any change in defined frequency allocation scheme requires consensus-based decisions at international level, which are associated to timeframes of decades and obviously difficult to accomplish. At national level, the modifications are less bureaucratic and potentially not so complex to materialize. The overall global framework for the use of spectrum, governed by International Telecommunication Union (ITU) Radio Regulations (RR), is foreseen to be quite stable, specifically regarding MMS allocations. Therefore, in the presented discussion, it is assumed that will be no significant changes at international level. However, it is expected that national frameworks might evolve towards a more dynamic management approach, which is in the foundations of the following reflexion.

Considering that, none of the $156-174 \mathrm{MHz}$ channels are exclusively allocated to MMS, each NRA can choose which service(s) to license in the band, given that the degree of compatibility between services shall be taken into consideration to minimize harmful interference. Moreover, NRA can allow frequency bands to be used for other purposes, providing that does not cause harmful interference to any service that is operating under an allocation in the RR. Under those circumstances, it is possible to conceive different ways of doing business and anticipate a change in national regulatory paradigms. Actually, FCC published a new framework [3], which abolished the actual spectrum management model in large parts of spectrum, and evolved towards a dynamic 
model. Thus, in the present discussion we pretend to address the required changes in regulatory framework that would answer to maritime community demands for broadband services and support emerging technologies and information exchange requirements. Additionally, it is important to discuss the associated opportunities and challenges of such intention and present an evolutional model to implement it.

\subsection{New Policy Definition}

The change in regulatory paradigm is an essential transformation to enable the deployment of DSA and an inevitable step towards mitigation of spectrum scarcity problems. However, the simple definition of a dynamic access based principle is not enough to characterize a governance model. For instance, interference control is a complex endeavour that needs to be enforced to ensure protection of primary systems. Eventually, the regulatory foundations need to be revisited and the fundamental objectives readdressed, to evaluate different possibilities to fulfil requirements, accommodate user demands and promote industry innovation, as represented in Figure 3.

The basis of a flexible regulatory environment, which might be able to accommodate dynamic utilization of spectrum, must include a well-defined command and control concept with two components: policy and enforcement. A spectrum access priority policy should translate the idea of agility and temporary allocations into a policy and rules that specify the way (allowed) users get access to the spectrum. Generically, the eligible techniques to rule spectrum access are priority and contention based. In a priority based spectrum access control, the users are classified according to their privileges to explore spectrum: incumbents (or primary) are frequency band assigned users, while opportunistic (or secondary) users are unlicensed users that are allowed to use allo-

\section{Back to Foundations...}

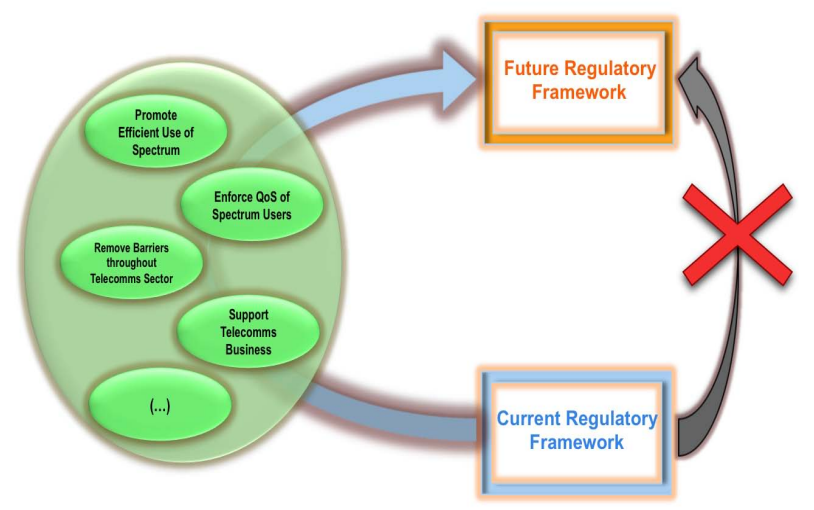

Figure 3. Paradigm change requires an evolutionary process that might need to re-address regulatory foundations to progress towards a new framework. cated spectrum as long as they do not harmful interfere with on-going operations of primary users. In a contention based access control, all the users have the same priority to get access to spectrum and compete for the resources (eventually) at same time. Mixed options are, of course possible, with contention being used within privilege classes. Complementary, this policy might have an evolution process that goes from exclusive use and interference free spectrum to priority based shared spectrum and mitigation of interference to appropriate QoS levels. The second component of command and control model is an enforcement program that is addressed later on this paper.

Another key issue is the definition of cooperation level between primary and secondary users. The general principle considers that secondary unlicensed users are allowed to use assigned spectrum, as long as they do not interfere with primary users, but it is not explicitly assumed any kind of cooperation. The underlay paradigm, where primary and secondary users do not cooperate, has the merit of independence and permits a shorter deployment time, being usually considered a natural choice. However, in the case of multiple secondary users, it does not incorporate any means to control that aggregated interference generated is kept below a certain threshold, since primary receivers are passive and may be located in the vicinity of secondary transmitters. Alternatively, one may include some sort of symbiotic cooperation where, for instance, primary user trades the relaying of its transmissions by secondary operation [4] or some other type of cooperation within overlay paradigm. In any case, the choice of spectrum sharing strategies can have a significant impact in the overall spectrum efficiency, but it also implies different level of cognition and implementation complexity, which leads to different challenges, as well.

Despite the obvious advantages at efficiency level, the evolution of regulatory framework, in the case of MMS, presents additional opportunities regarding change management and field deployment. In MMS, most of frequency bands are assigned to a service, instead of a specific user. It is common to have multiple users getting access to a frequency in a maritime band, without any kind of precedence or supervising in a contention type of scheme. In fact, such situation constitute, in practice, some sort of early deployment of DSA similar concept, which constitutes an opportunity for a new paradigm transition phase. Considering that disruptive processes need transition plans and evolution strategies that are not compatible with "big bang" approaches, any migration process should promote a smooth evolution without disturbing current operations or delaying start-ups based on new concepts. In this context, a transition plan to introduce new practices in MMS bands would be highly 
simplified.

\subsection{Enforcement Mechanism}

In addition to spectrum access priority policy, it is essential to establish a course of action to assure its enforcement, which naturally includes a set of metrics, procedures and organizations to guarantee their practical implementation. Harmful protection does not necessary means interference abolition, providing that communications link conditions are appropriate to ensure the required QoS. In other words, interference is allowed whenever it does not affect the fulfilment of the minimum requirements for service provision. Conceptually, the incumbent QoS assurance is built on the basis of a trustful relationship between primary and secondary users in a shared spectrum venture. In the past, the fear of harmful interference has resulted in the exclusiveness of spectrum usage for most of bands and restrictions on out of band interference. The QoS was guaranteed through a regulatory policy enforcement based on auditing practices, which were supposed to guarantee that only assigned users could use the corresponding frequency bands. The focus of QoS assurance was definitely on interference abolition. In the future, the new spectrum access paradigm will change the focus towards maximization of usable capacity. Depending on the communications service type, different Signal-to-Noise-plus-Interference-Ratio (SNIR) may result in the same system performance. In other words, interference free and concurrently usage of spectrum (with acceptable levels of interference) may produce the same practical results, in terms of incumbent service provision QoS, but with obvious differences in terms of spectrum efficiency. The key point is the definition of a service interference tolerance that needs to be preserved.

Similarly to classical regulatory framework, a policy definition and a set of well-defined rules are not enough to guarantee the interference at proper levels. The enforcement is critical to guarantee spectrum access, in compliance with allocation and assign criteria, and important to overcome user's concerns. Even so, in the case of DSA, the fact that interference is allowed, as long as it does not compromise the incumbent's system performance, post an additional challenge, which make a huge difference in auditing operations and on harmful interference verification and enforcement, as summarized in Figure 4. It is critical to come up with a framework that can be able to handle this new way of doing business, specifically, it is essential to address practical aspects that are fundamental for the effectiveness of regulatory authorities in their spectrum policy execution. Recently, FCC Spectrum Policy Task Force [5] has recommended a paradigm shift in interference assessment, introducing a new metric, called interference temperature, which is

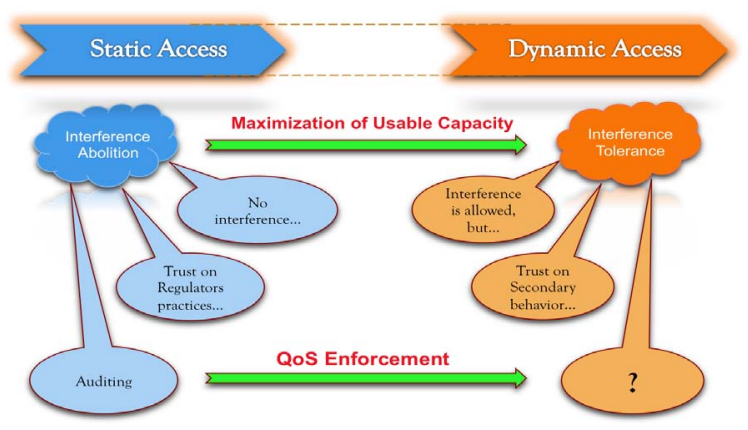

Figure 4. In the new paradigm, what will be the corresponding auditing operations.

intended to quantify and manage the sources of interference in a radio environment. The idea is to characterize a worst-case scenario, where a primary receiver is able to support the defined QoS and specify the interference temperature limits that cannot be exceeded [6], for each frequency band. Eventually, such approach, conceptually interesting, might be difficult to apply to real systems, without any further developments. In any case, normalization and standardization requirements, such as reference values, thresholds and metrics, are critical to create a common understanding of criteria for operational requirements, spectrum access conditions, users' relationships and regulatory authority roles.

Additionally, in a DSA environment, where users rights to transmit are distinctive and spectrum access control is decentralized, the policy enforcement based on spectrum auditing can be really challenging. From a regulatory perspective, it is critical to be able to perform identification, classification and localization of spectrum users to control interference levels, assure incumbents rights and conciliate demands. Those capabilities are not trivial to implement and continuously support, because in most cases, opportunistic users are difficult to register and log. Therefore, in addition to inevitability of metrics definition, regular policing of spectrum use would benefit from prior to market entry equipment certification. Such procedure would endorse metrics fulfilment compliancy mitigating the probability of unintentional interference and facilitating spectrum use policing.

\subsection{Transition Plan}

Considering the general constraints and opportunities associated to spectrum management paradigm change and the technological state of the art, the incremental spiral approach is definitely the appropriate strategy for an evolution of regulatory framework. Not only because a migration of this nature cannot be performed in one step, but also due the maturity level of enable technology, stakeholders, regulation and standardization. This process has to be performed through incremental steps of 
functionalities, where each stage should be carefully is designed, implemented, tested and evaluated. Furthermore, new iterations have to be planned, taken into consideration the lessons learned from previous phases, and according to the level of technological maturity and expansion of applied spectrum.

Initially, a pilot implementation is a good approach to large-scale proof of concept. For instance, a set of field tests might be performed in VHF channels assigned to new technologies. Furthermore, the process can be expanded to the opportunistic use of spectrum on carefully selected non-critical analogue voice assigned channels. Actually, this manoeuvre does not present significant risks for incumbent operations, even in early stages of cognition maturity of secondary radios, because analogue voice services have a good tolerance to noise and interference. Nevertheless, pilot testing need to be carefully documented, considering comprehensive communication and change management plans, to ensure that all stakeholders would positively impact the process. This methodology would permit quick wins and potentiate concepts consolidation, increasing users' confidence in process development.

Subsequently, a set of initial spirals has to be planned, each one with an associated cognitive (radio) maturity level and applied portion of spectrum, as represented in Figure 5. The development governance has to be performed, taken into consideration the practical results of each spiral, because it requires large-scale implementation testing, which is difficult to emulate in laboratory. Eventually, at low levels of cognition, the deployments may include beacons to help the searchers and some sort of "spectrum access on demand" based on geo-location and databases to find white spaces in the area. Subsequently, new cognition functionalities, with detection algorithms more capable and complex, could be deployed and validated.

\subsection{International Coordination}

The envisage maritime B-VHF networks can potentially

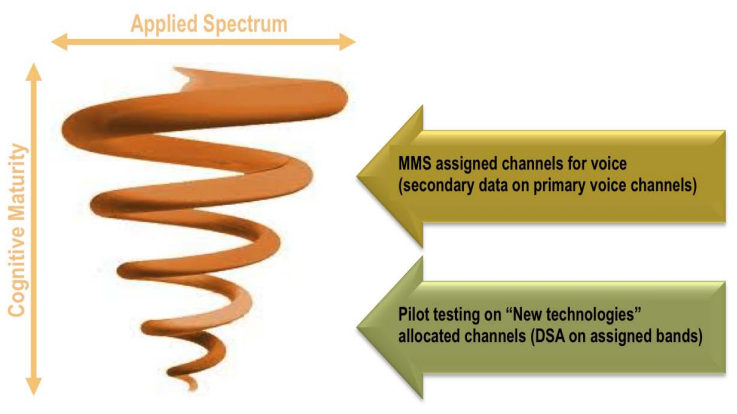

Build a little, test a little...

Figure 5. Incremental spiral approach to evolution of regulatory framework. be implemented all over the world. Nevertheless, the scope and applied spectrum might vary between countries. Actually, these do not constitute a problem, from regulatory perspective, since MMS assigning channels is a NRA responsibility. The only aspect that needs to be taking into consideration is the fact that some ships may need to roam between B-VHF networks operated from different countries. In this case, it is necessary to implement a control mechanism to support roaming, which can be conceptually similar to cellular communications implemented solutions. Anyway, international coordination is required to avoid disturbances in spectra, from adjacent countries, that are not applying the same concepts to common frequency bands.

Furthermore, regulatory policy coherence is highly recommended, not only to harmonize and combine QoS enforcement, but also to facilitate technological developments and equipment interoperability. These efforts would also potentiate operational issues, regarding system's exploration and support, namely prevention of harmful interference and guarantee of incumbent's rights, within overlapping footprints of different nations. Complementary, it might also be consider to include such international coordination within ITU activities, similarly to other frequency bands, like high frequency, given the potential of maritime B-VHF networks to have transnational coverage and roaming between nationally explored networks.

\section{Deployment Challenges}

The major challenge for a DSA strategy deployment is definitely the QoS enforcement. Firstly, it is necessary to build-up a common understanding criteria for the definition of operational requirements, spectrum access conditions, (incumbents/opportunistic) users relationships and regulatory roles. Additionally, it is crucial to ensure that policy is implemented and followed. Moreover, the maximization of usable capacity is in the basis of opportunistic use, changing paradigm from interference abolition to interference tolerance. The consequences of such transformation, associated to an inevitable decentralized spectrum control access and an unavoidable difficulty to register and log opportunistic users have also a significant impact on QoS enforcement practices and procedures. Such challenging context needs to be characterized in terms of performance of each stakeholder in order to be represented with adequate metrics that would allow spectrum survey and user's scrutiny, as summarized in Figure 6.

Dynamic and unpredictable environments are not simple to characterize, particularly in terms of evaluation metrics. In a CR based DSA scenario there are basic operational areas that need to be addressed, namely primary and secondary utilization of spectrum and their relation- 


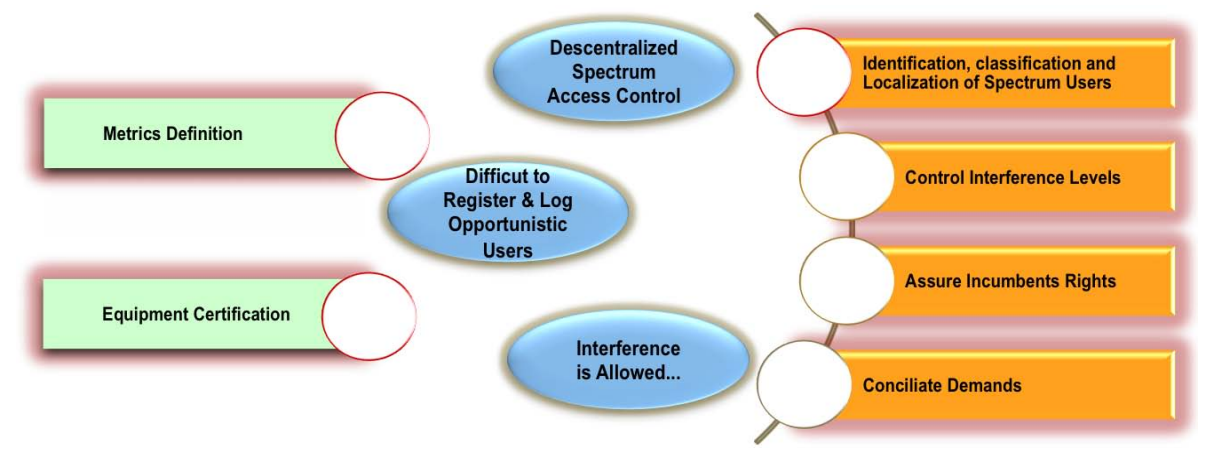

Figure 6. Deployment challenges of dynamic management strategy.

ship. On primary side, the Acceptable Level of Interference (ALI) depends upon primary receiver sensitivity value, communication service and its respective QoS. Therefore, it is necessary to characterize and quantify each one of those requirements and find the appropriate metrics to relate them and their effect on interference level. Furthermore, on the secondary side, the most critical issues are the spectrum sensing capability, its implementation and performance. Moreover, it is not only the detection of primary activity, its start and stop momentums, but also the monitoring any primary activity while secondary is using the spectrum (i.e. secondary should implement sensing periods between transmissions to detect eventual primary operation). The evaluation metrics of such performances and its practical application to real systems are critical to prevent harmful interference and assure incumbents rights. Finally, it is necessary to detail interactions, procedures and grading between primary and secondary users and between secondary's themselves, the later depends upon spectrum access policy.

\subsection{Primary Users’ Figures of Merit}

In a context of opportunistic use of spectrum, metrics such as reference values and thresholds are very important, not only to establish a common understanding criteria for spectrum users coexistence, but also to support regulatory roles enforcement. Concepts such as operational requirements, spectrum access conditions, primary/secondary users relationships and regulatory authority roles need to be translated in values that can be measure and compared in order to enforce spectrum management policy and assure service level requirements. The QoS enforcement is a clincher to control interference levels, assure incumbents rights, conciliate demands and resolve disputes. Actually, this condition is essential for incumbents' support to any evolution towards DSA and opportunistic use of spectra, given that their live operations depend on ALI assurance. Inevitably, the fact that spectrum might be used in opportunistic basis has potential impact on incumbent systems, which might limit services performances. Hence, such possibility needs to be addressed and quantified in metrics that allow an evaluation of interference harmful protection.

The practical consequences of inappropriate secondary activity are noise floor level rising and an increase in service outage probability, which directly restricts incumbent's achievable capacity. The threat, for primary service, might intensify as a consequence of cumulative noise and risk of interference, depending on the amount of secondary service users within coverage area of primary network. Essentially, this effect is due to aggregate interference and to increase on probability of miss detection in secondary spectrum sensing, which results in secondary transmission during incumbents operation and rising on outage probability of live services. Hence, inaccuracy in spectrum sensing process is probably the major cause of disturbances in performance of primary systems. Anyway, the consequences of such inconvenient events depend upon the incumbent services' ALI, which is a function of users activity (duty cycle), service operating requirements and Receiver Operation Characteristics (ROC).

In an interference tolerant context, it is necessary to characterize each incumbent minimum operating requirements, namely its availability and reliability, in order to support the QoS assurance process. Unfortunately, most of MMS assigned bands (if not all) have no explicit operational requirements (QoS) for supported services, probably because it is implicitly considered best effort reached within an interference free environment. In the future, this aspect needs to be rectified upfront. Depending on type of service, and its associated performance demands, the ALI might vary. For most of MMS services, typically analogue voice, the operating requirements are quite permissive, but in the case of digital services, such as DSC and AIS, the admissible interference constraints are more restrictive. Obviously, primary receiver's probability of miss detection and probability of false alarm, characterized in ROC, are critical for systems behaviour, especially under lower SNIR. Finally, in addition to miss detection probability, aggregate interference is also a function of primary users activity, which, in 
MMS, is quite atypical. Depending on assigned band, even for the same type of service, the traffic profile might be completely different. For example, channel 16 has duty cycle quite different from channel assigned for recreation sailing voice communications. Nevertheless, all of those effects and consequences need to be related with primary's QoS requirements, specifically Service Level Requirements (SLR).

Thus, the challenge is to express incumbent's SLR into metrics that would be able to support primary QoS enforcement and facilitate opportunistic systems design. The SLR, which are typically specified in terms of availability, reliability and performance, need to be translated into metrics that, ultimately, point to an ALI that would be measured and enforced. Hence, the natural course involves operational conditions managing by controlling SNIR level that would allow the incumbents service to operate according to SLR. On the other hand, service outage probability and achievable capacity are assenting ways to evaluate the consequences of secondary activity. For both, the assessment is based on the analysis of radio environment conditions and its ability to fulfil service requirements. In other words, these examinations pretend to verify how often the SNIR at primary receiver is below a certain threshold. Apparently, SNIR would be the natural metric to assure ALI and SLR. However, the problem is that SNIR might not be enough to characterize SLR. Eventually, it can be representative of primary service performance, but it is not sufficient to give evidence of availability and reliability requirements, due to lack of time dimension characterization. Therefore, ALI might need to incorporate, not only interference limitations, but also a time domain component that might be able to express the environment dynamics.

\subsection{Secondary Users' Figures of Merit}

The purposes of secondary users related metrics are slightly different from the incumbents'. While primary services metrics are associated to regulatory policy enforcement, the secondary service metrics pretend to measure the ability of secondary users (systems) to fulfil regulatory roles and act as indicators of feasibility and effectually of deployments. The success of opportunistic systems depends on their ability to exploit inactive frequency bands. Specifically, it depends upon secondary's capability to find the so-called opportunities to operate and consequently on its spectrum sensing techniques capabilities. Therefore, the capability to detect and classify spectrum holes, within accurate levels of spectral resolution, to estimate the direction of arrival of interferers and to do it in real time, is definitely a critical asset. On the other hand, there are radio environment characteristics that determine operating conditions of opportunistic systems, independently of its own performances, namely detection sensibility and processing or incumbent's activity profile (duty cycle). Ultimately, these constraints would limit achievable performances in such a way that secondary deployment might be not interesting and/or rewarding. Hence, there are two categories of metrics for secondary services that need to be addressed and quantified, which may be generically designated as compliance and performance metrics.

The harmful interference depends on secondary capacity (and behaviour) to detect primary activity and carry out spectrum sensing while opportunistically uses the spectrum. The primary activity detection requires a spectrum sensing algorithm that, not only be able to detect the presence of primary transmissions and overcome the effects of attenuation, fading and shadowing, but also to perform it in appropriate time frames in order to maximize the spectrum holes achievable capacity. Specifically, it is critical to detect, as accurate as possible, the start/ stop momenta of primary transmissions. Therefore, the minimization of harmful interference implies some sort of normalization with reference values for detection performance and sensing periods between secondary transmissions. In any case, it is not simple to define a benchmark for detection performance, but it is more difficult to come up with spectrum sensing evaluation metrics.

Given the multiplicity of methodologies to deal with spectrum holes detection, each one presenting advantages and drawbacks, it is essential to quantify the required detection performance due to its relationship with the safety granted to primary users. Digital radio receivers have been characterized by a curve that plots probability of miss detection as a function of probability of false alarm, for a fixed sensing time and operating signalto-noise ratio. This means that detectors performance is traditionally associated to the evaluation of sensitivity, probability of miss detection and probability of false alarm [7]. However, the outcome of traditional metrics refers to a complete system model, because sensitivity and probability of miss detection are related with a protection level of primary user while probability of false alarm is related with the secondary user performance. If the focus is sensing performance evaluation itself, an assessment at system level might not be useful. This issue needs to be addressed with an abstraction level that might be able to characterize performance of spectrum sensing solution and quantify it for comparison purposes. Obviously, that is not simple to achieve and probably it is necessary to find indirectly ways of measuring sensing behaviour.

Another challenging issue is the definition of a reference criterion for sensing periods between secondary transmissions. Naturally, spectrum sensing solution needs to accommodate the fact that spectrum holes have 
a dynamic nature, particularly in time domain. In other words, incumbents activity might be initiated anytime, so secondary users need to continuously perform spectrum sensing, even between opportunistic transmissions. Again, it is not easy to normalize the sensing periods required for supported services and incumbent harmful interference avoidance. In brief, the described spectrum sensing metrics are important to evaluate the secondary systems ability to exploit inactive frequency bands, without disrupting primary live services. This assessment does not necessary means a prior to operate authority inspection, or similar, but definitely would be highly supportive of QoS enforcement operations.

The maximum achievable capacity of an opportunistic network is able to summarize the interest and incentive for its deployment, acting as a single benchmark for viability analysis. The secondary systems performances depend not only on its own faculty to exploit spectrum holes, but also on spectrum holes occurrences and characteristics. As previously mentioned, spectrum sensing algorithms shall be able to detect primary start/stop transmissions instants, as soon as possible, to minimize the harmful interference and maximize time of opportunistic usage. On the other hand, channel state estimation play an important role in situation analysis, providing information to define spectral availability and channel characteristics, like noise floor statistics and channel capacity, allowing dynamic adaptation and/or reconfiguration of transmitted signal to maximize the throughput of the link. In the learning process, it is crucial to detect spectrum holes, estimate their power contents, and predict its availability to support high reliable communications. Thus, radio scene analysis includes the detection of spectrum holes and the evaluation of noise floor and traffic statistics that will be used as inputs for adjustments in power and spectrum management. Additionally, it is required another input, which provide an estimation of channel capacity and allow coherent detection. Nevertheless, incumbent's traffic profile characteristics, namely transmission time and duty cycle, might preclude opportunities in such a way that it is not possible or effective to use them. For instance, primary bursty traffic, with short transmission time and small times between transmissions might be useless at all. In conclusion, radio scene analysis competence will determine the achievable capacity of a secondary network, which ultimately is decisive for its deployment decision.

\subsection{Equipment Certification}

Trust is the foundation of primary-secondary users' relationships in a DSA based scenario. Obviously, this confidence conception needs to be regulated and enforced, not only because secondary users require guidance and assistance to avoid harmful interference, but also because incumbents demand for support of their live operations QoS. One potential enabler for interference handling, and consequently, a front line for the implementation of a sureness aptitude would again be equipment certification, prior to its market entry. Understandably, the probability of unintended harmful interference is clearly mitigated, when a certified secondary system pretends take advantage of opportunistic spectra. In fact, incumbents QoS enforcement may start with a compliance verification of secondary systems.

However, the establishment of a worldwide certification capacity requires an international level of coordination, which might be equivalent, in complexity, to spectrum management processes. There are well known industry joint ventures and non-profit organizations that have been proved to be successfully in their missions of connectivity, interoperability and quality assurance of enabled products. Wi-Fi Alliance [8] is probably one of the most successful experiences in this area. However, those types of association based certification initiatives and their driving effects are highly dependent upon the amount of members, their industry footprint and the amount of deployed systems/equipment. In the case of maritime B-VHF communications, probably neither the amount of members, nor its industry footprint would be a problem. Eventually, the drawback of a Wi-Fi Alliance similar initiative, for maritime VHF, would be the potential amount of target terminals. Nevertheless, and even though a hypothetical worldwide equipment certification would not substitute the need for auditing operations and other policy enforcement initiatives, it would definitely be an importance complementary tool.

\section{Concluding Remarks}

The practical interest of maritime VHF based broadband services is easily recognizable and so is spectrum unavailability, either in MMS assigned spectrum or VHF spectrum in general. DSA and CR are pointed as decisive strategies to increase efficiency of electromagnetic radio spectrum usage and overcome its scarcity. Naturally, the expected efficiency improvement is proportional to the level of cognition functionalities available in a CR, but enabling technologies for maritime B-VHF communications are in the basis of some of the most important telecommunications infrastructures, such as cellular networks, and are topics of intensive research. The journey is definitely challenging, so every step should be carefully considered and based on criteria of feasibility, support and coexistence with legacy systems. However, similarly to other technological achievements, maritime B-VHF may end up in a situation where technological progress is far more intense than its equivalent in regulatory policy and plans. 
Definitely, currently regulatory framework is not only inadequate for emerging radio communications concepts and technologies, but also a potential barrier to its deployment. It is necessary to evolve towards a more flexible and dynamic approach to spectrum management, comprehensively preparing the transition phase and involving stakeholders. The path from exclusive use and interference free spectrum towards priority based shared spectrum and mitigation of interference to appropriate QoS levels is complex, but is essential to support users' needs and increasing demand for information exchange. Vital aspects such as QoS enforcement and operational livelihood depend upon the definition of adequate metrics and processes that goes from auditing activities to eventual equipment certification. The role of regulatory agencies is crucial in this process, to promote discussions, mediate processes and support solutions. The regulatory paradigm shift is inevitable, so the question is how its agenda will impact the systems under development.

The presented analysis pretends to contribute for discussion on the real need for regulatory framework evolution, specifically in maritime VHF bands, focusing the arguments on the solution, instead of the problem. The paradigm change encompasses a new policy definition, an enforcement mechanism and a transition plan. For all of those constituents it is necessary to identify the appropriate solution and the way to implement it, taking into consideration the existing environment. In the current analysis, we have been centred in the identification of challenges and opportunities associated to the maritime environment, addressing potential tracks for further investigation. It is now necessary to evaluate the feasibility of such courses of action and hand up with specific solu- tions for each case.

\section{REFERENCES}

[1] J. Mitola III, “Cognitive Radio: An Integrated Agent Architecture for Software Defined Radio," Dissertation, Computer Communication System Laboratory, Department of Teleinformatics, Royal Institute of Technology (KTH), Stockholm, 2000.

[2] Federal Communications Commission, "Notice of Proposed Rulemaking and Order: Facilitating Opportunities For Flexible, Efficient, and Reliable Spectrum Use Employing Cognitive Radio Technologies,” ET Docket No. 03-108, 2005.

[3] Federal Communications Commission, "Promoting Efficient Use of Spectrum through Elimination of Barriers to the Development of Secondary Markets," Report WT Docket No. 00-230, 2003. http://www.fcc.gov/sptf/

[4] L. Giupponi and C. Ibars, "Distributed Cooperation in Cognitive Radio Networks: Overlay Versus Underlay Paradigm," Vehicular Technology Conference, Barcelona, 26-29 April 2009, pp.1-6.

[5] Federal Communications Commission, "Spectrum Policy Task Force,” Rep. ET Docket No. 02-135, 2002.

[6] S. Haykin, "Cognitive Radio: Brain-Empowered Wireless Communications," IEEE Journal on Selected Areas in Communications, Vol. 23, No. 2, 2005, pp. 201-220. http://dx.doi.org/10.1109/JSAC.2004.839380

[7] S. M. Kay, "Fundamentals of Statistical Signal Processing: Detection Theory," Vol. 2, Prentice-Hall, Upper Saddle River, 1998.

[8] www.wi-fi.org 